\title{
Modulación del estado de ánimo a través de estímulos musicales activantes. Un diseño experimental con adultos jóvenes
}

\author{
Modulation of mood by activating musical stimuli.
}

An experimental design with young adults

Candela Castro ${ }^{1}$, Veronika Díaz Abrahan ${ }^{2}$ y Nadia Justel ${ }^{3}$

\begin{abstract}
${ }^{1}$ Licenciada en Musicoterapia. Buenos Aires, Argentina. E-mail: castrocande@gmail.com ${ }^{2}$ Licenciada en Musicoterapia. Doctora en Neurociencias. Becaria doctoral del Consejo Nacional de Investigaciones Científicas y Técnicas (CONICET) en el Laboratorio Interdisciplinario de

Neurociencia Cognitiva de la Universidad Nacional de San Martín, Buenos Aires. Docente

de la Licenciatura en Musicoterapia de la Universidad de Buenos Aires (UBA). E-mail: abrahanveronika@conicet.gov.ar

${ }^{3}$ Doctora en Psicología. Directora del Laboratorio Interdisciplinario de Neurociencia Cognitiva de la Universidad Nacional de San Martín, Buenos Aires. Investigadora Adjunta del CONICET.

E-mail: nadiajustel@conicet.gov.ar

Licenciatura en Musicoterapia, Facultad de Psicología, Universidad Nacional de Buenos Aires (UBA).

Buenos Aires, Argentina.
\end{abstract}

\section{Resumen}

La música provoca respuestas emocionales que se ven reflejadas en los cambios fisiológicos y cognitivos de los oyentes. Es una temática de estudio en común entre distintas disciplinas como la psicología de la música, la musicoterapia, la antropología de la música y la la neuropsicología, entre otras. Entender de qué manera la música puede incidir en el estado de ánimo de las personas permite contribuir al conocimiento sobre el efecto comportamental y a la creación de nuevas técnicas orientadas para regular las emociones mediante la música. Teniendo en cuenta estos antecedentes, se realizó un estudio con el propósito de evaluar el efecto de la percepción de una pieza musical con características activantes (grupo experimental) versus ruido blanco (grupo control) como modulador del estado de ánimo, desde la perspectiva de las neurociencias, contando con la participación de adultos de entre 18 a 40 años. La inducción emocional de los participantes se realizó a través de la observación de imágenes con valencia emocional negativa. Posteriormente, y bajo una distribución al azar, un grupo de voluntarios escuchó la pieza musical activante y otro grupo escuchó el ruido blanco; ambos estímulos auditivos duraron 3 minutos. Se evaluó el estado emocional antes y después de la percepción auditiva. Los resultados analizados a través de pruebas no paramétricas (test de Wilcoxon) indicaron que la música con características activantes moduló emociones de tristeza y ansiedad, disminuyéndolas significativamente. Estos hallazgos sugieren que la percepción musical presenta potencialidades para la reducción del estrés, así como la ansiedad, brindando evidencia desde un enfoque neurocognitivo.

Palabras clave: percepción musical, estado de ánimo, modulación, emociones, neurociencia cognitiva

\section{Abstract}

Music, from its several possibilities, induces emotional responses, which are reflected in the 
physiological and cognitive changes of the listeners, this is a thematic of study in common among different disciplines: music psychology, music therapy, music anthropology, neuropsychology, among others. At the neuroanatomical level, neuroimaging studies have shown that music can modulate the activity of limbic and paralimbic structures, that is, structures that are especially involved in the initiation, generation, maintenance, completion and modulation of emotions. Being able to understand how music can affect the mood of people allow us to contribute to the creation of new techniques oriented to emotional regulation through music. The current study was develop with the aim of evaluating the effect of perception of a musical piece, with activating characteristics (experimental group) vs. white noise (control group), as mood modulator, from a neurosientific perspective. Participated 29 young adults (55\% man), between 18 to 40 years old ( $M=$ $22.07, S D=.43)$, with no presence of neurological diseases or amusia, nor consumption of medications at the time of the study. The selection of the musical piece with activating characteristics was evaluated in a preliminary study, which counted with the participation of 24 young adults, who valued three pieces of music in terms of valence (emotion that the piece generated) and arousal (activation level). The 3 pieces were: "Tika Tika Walk" by Carlos D'alessio; "Leafmen" by Danny Elfman and "Define Dancing" by Thomas Newman. From this preliminary study, the activating stimulus that would be used for emotional modulation was selected ("Leafmen" by Danny Elfman). The emotional induction of the participants was carried out through the observation of 24 images with negative emotional valence (extracted from International Affective Picture System). About procedure, the study consisted of a single session of activity, divided into the following five phases: (1) information phase: participants signed the informed consent and completed the socio demographic questionnaire, (2) emotional induction phase: participants observed 24 negative images, and they had to rate each image from 0 to 10 (not arousing/ emotional at all to highly arousing/emotional), (3) pre- measurement phase: participants completed a scale of mood, instrument used to measure transient moods, (4) perception phase: under a random distribution, a group of volunteers listened the activating music piece and another group listened the white noise (both for 3 minutes), and finally (5) post-measurement phase: participants completed a scale of mood after music or white noise perception. We analyzed the assumptions of normality and homogeneity of the sample using the statistics of Levenne and Shapiro Wilk. The results analyzed through non-parametric tests (Wilcoxon test, with a $p$ value of .05). In the premeasurement of mood, no significant differences were found between groups. Results of post- measurement phase indicated that music with activating characteristics, modulated the emotions of sadness and anxiety, decreasing them significantly. The results obtained indicate that music, specifically selected, aided as a modulator of emotions. It is important not to generalize the results obtained to the whole population, in this sense and thinking in future research, it would be necessary to expand the sample to a larger $n$, as well as to investigate this effect in other samples. The results obtained allow to open new paths in terms of the intrinsic qualities of music and its elements to promote relief of anxiety and stress, regulate moods and, promote motivation or enable the development of mechanisms to adapt to situations new and/or traumatic. Finally, it could be assumed that music constitutes an effective stimulus to modulate emotional responses, which are reflected in the mood changes of the listeners.

Keywords: musical perception, mood state, modulation, emotions, cognitive neuroscience

\section{Introducción}

La música constituye un estímulo efectivo para provocar respuestas emocionales que se ven reflejadas en los cambios fisiológicos 
y cognitivos de los oyentes (Koelsch, Skourasy Lohmann, 2018). Varios especialistas en música, neurociencias y psicología de la música, entre otras disciplinas, han investigado acerca de la relación existente entre la música y las emociones (Blood y Zatorre, 2001; Diaz, 2010; Juslin y Sloboda, 2010).

Hay dos principales posturas que explican la evocación de emociones a través de la música: la cognitiva, según la cual las emociones producidas por la música dependen directamente de las experiencias previas de las personas y de las asociaciones producidas entre el estímulo emocional y el contexto en que se presenta y, por otro lado, la emotivista, según la cual las emociones producidas por la música se deben específicamente a sus características propias (Radford, 1989). Esta última línea se ha reforzado a lo largo de los años a través de estudios experimentales, los cuales comprueban que la música produce diversos efectos fisiológicos como, por ejemplo, la modificación en el ritmo cardíaco, la frecuencia respiratoria, la presión arterial, la conductancia de la piel, la temperatura corporal y las respuestas bioquímicas (Altenmüller y Schlaug, 2015; Chanda y Levitin, 2013; Fancourt, Ockelford y Belai, 2014; Peretz, Gagnony Bouchard, 1998), con una activación constante de la amígdala, independientemente de la emoción que provoque (Gosselin, Peretz, Johnseny Adolphs, 2007; Peretz et al., 1998). Estos resultados sugieren además que, en general, los estímulos musicales evocan una respuesta emocional específica y genuina en los oyentes, aunque algunos despiertan un grupo de emociones concretas (de valencia positiva, por ejemplo) y otros una respuesta más general (por ejemplo, ser agradables e intensas al mismo tiempo; Flores-Gutierrez y Díaz, 2009).

Actualmente la música se usa cotidianamente para influir directa o indirectamente sobre el estado emocional, modular momentáneamente la atención y la concentración e inducir sostener relaciones sociales (Juslin y Västjfäll, 2008; Saarikallio y Erkkilä, 2007; Sloboda y O’Neill, 2001; Von Georgi y
Polat, 2013) o incidir sobre la formación de la memoria (Justel, O'Connor y Rubinstein, 2015). Las personas con alta susceptibilidad emocional usan la música más frecuentemente para influenciar emociones y situaciones afectivas (Chamorro-Premuzic y Furham, 2007).

A nivel neuroanatómico, los estudios de neuroimagen han mostrado que la música puede modular la actividad de estructuras límbicas y paralímbicas, es decir, estructuras especialmente involucradas en la iniciación, generación, mantenimiento, finalización $\mathrm{y}$ modulación de las emociones (Blood, Zatorre, Bermúdez y Evans, 1999; Blood y Zatorre, 2001; Diaz Abrahan, Fisher y Justel, 2017; Gosselin et al., 2005; Koelsch et al., 2006), lo que sugiere que diversos estímulos musicales podrían regular las emociones, a través de la influencia que ejerce la música sobre la activación neuroanatómica vinculada a la emoción.

Estos hallazgos son el sustento teórico para avalar tratamientos con música en desórdenes afectivos como la depresión, la ansiedad o el estrés postraumático, ya que son trastornos, en parte, relacionados con la disfunción de estructuras límbicas, como la amígdala, y paralímbicas, como el córtex órbito-frontal (Koelsch, 2009). La regulación emocional puede involucrar tanto la mantención y el realce de la emoción como, también, su inhibición o supresión, implicando tanto estrategias adquiridas de automanejo emocional (Silva, 2005; Thurston, Bell y Induni, 2018), como también influencias externas, por ejemplo la música (Koelsch et al., 2018).

En el contexto de estos antecedentes, el objetivo del presente trabajo fue evaluar si la percepción musical sería una herramienta efectiva para regular el estado de ánimo de adultos jóvenes, a través de un diseño experimental. Se espera que la audición de un estímulo musical específicamente seleccionado genere una mejora en el estado emocional negativo de los sujetos, el cual será inducido a través de la observación de imágenes con contenido negativo. 


\section{Método}

\section{Participantes}

Se contó con la participación de 29 sujetos (55\% varones), de entre 18 a 40 años $(M=$ $22.07, D E=.43)$, sin presencia de enfermedades neurológicas o amusia, ni consumo de medicamentos por enfermedad crónica al momento del estudio (aspecto que se incluyó como criterio de exclusión para trabajar con una población sana). El trabajo se rigió de acuerdo a las normas éticas vigentes (Asamblea Médica Mundial, Declaración de Helsinki, 2013). Se les solicitó a los participantes que firmasen un consentimiento informado en el que se detallaban los propósitos del estudio y el compromiso de asegurar el anonimato y la confidencialidad de los datos. Además, se les informó que, si lo deseaban, podían abandonar el estudio en cualquier momento de su realización. Luego de la firma del consentimiento informado, los participantes completaron un cuestionario en el que se solicitaba información socio-demográfica (con datos básicos de edad, sexo, ocupación, consumo de sustancias psicoactivas en la última hora, consumo de medicamentos y sustancias, antecedentes psiquiátricos, tratamiento psiquiátrico actual, para corroborar los criterios de exclusión de la muestra).

\section{Materiales}

\section{Inducción emocional}

Se utilizaron 24 imágenes del Sistema Internacional de Imágenes Afectivas (IAPS por su sigla en inglés, International Affective Picture System; Lang, Bradley y Cuthbert, 1995), con valencia emocional negativa. Las imágenes fueron seleccionadas de acuerdo a trabajos previos (Cahill, Gorski y Le, 2003; Diaz Abrahan, Shifres y Justel, 2018; Justel Y Ruetti, 2014). En una planilla que contenía 24 ítems, el sujeto, a medida que observaba las imágenes, debía indicar cuán activante le parecía cada una de ellas en una escala de 10 puntos (siendo 0 nada activante o emocional y 10 muy activante o emocional).

\section{Escala de Valoración del Estado del Ánimo (EVEA)}

La EVEA es un instrumento que sirve para medir estados de ánimo transitorios (Sanz, 2001). Consta de 16 ítems, cada uno compuesto por una escala gráfica de tipo Likert de 10 puntos (de $1=$ nada a $10=$ mucho), que presenta en su margen izquierdo una corta afirmación que describe un estado de ánimo. Las 16 frases tienen la misma construcción; todas empiezan con la expresión "me siento" y continúan con un adjetivo que representa un estado de ánimo (por ejemplo, "me siento triste", "me siento alegre"). La EVEA pretende evaluar cuatro estados de ánimo: ansiedad, ira-hostilidad, tristeza-depresión y alegría. A cada estado de ánimo le corresponden cuatro frases, quedando así definida una subescala para cada uno de ellos.

\section{Tratamientos}

\section{Condición experimental (Pieza musical)}

Inicialmente se evaluaron tres piezas musicales pertenecientes a bandas sonoras instrumentales para seleccionar aquella que sería empleada como moduladora del estado de ánimo (Tika Tika Walk del compositor Carlos D'Alessio, Leafmen del compositor Danny Elfman y Define Dancing del compositor Thomas Newman). Estas piezas fueron seleccionadas de acuerdo a las condiciones establecidas que definen a un estímulo activante: modos armónicos mayores, tempo acelerado, timbre estridente, ritmo con subdivisiones temporales, dinámica y/o forma que rompe con la expectativa del oyente (Grocke y Wigram, 2007; Kreutz, Ott, Osawa, Teichmann y Vaitl, 2008), que han demostrado que elevan los niveles de arousal (excitación / alerta) considerándose, por ende, activantes 
del sistema nervioso (Rickard, 2004).

Se puso a prueba la percepción de estas tres piezas (una duración de tres minutos cada una, reproducidas con una intensidad media) para corroborar que el estímulo a utilizar realmente fuese considerado una condición activante para el sujeto, recolectando información a través de una planilla en la cual los participantes debían otorgar un número de arousal que iba del 1 al 10 (consignado a través de la pregunta “¿Qué tan activante es?”, siendo 0 nada activante o emocional y 10 muy activante o emocional). En esta instancia participaron 24 sujetos diferentes a los que participaron en el protocolo de inducción y regulación emocional (18\% varones), de entre 18 a 40 años $(M=24.07, D E=1.22)$.
Se utilizó un análisis de varianza de una vía (ANOVA) para comparar las tres piezas musicales calificadas por los participantes. En relación al arousal (activación) los resultados pueden visualizarse en la Figura 1, en la que se observa que la pieza Leafmen fue la que calificaron como más activante. El ANOVA corroboró estas impresiones $F(2,80)=8.06 ; p$ $<.001$. Los análisis a posteriori indicaron que la pieza Leafmen se diferenció de Tika Tika y Define dancing $(p<.001)$, mientras que estos últimos estímulos no se diferenciaron entre sí $(p>.05)$. A partir de los resultados obtenidos, se seleccionó la pieza musical Leafmen para el estudio de inducción y modulación emocional del presente trabajo.

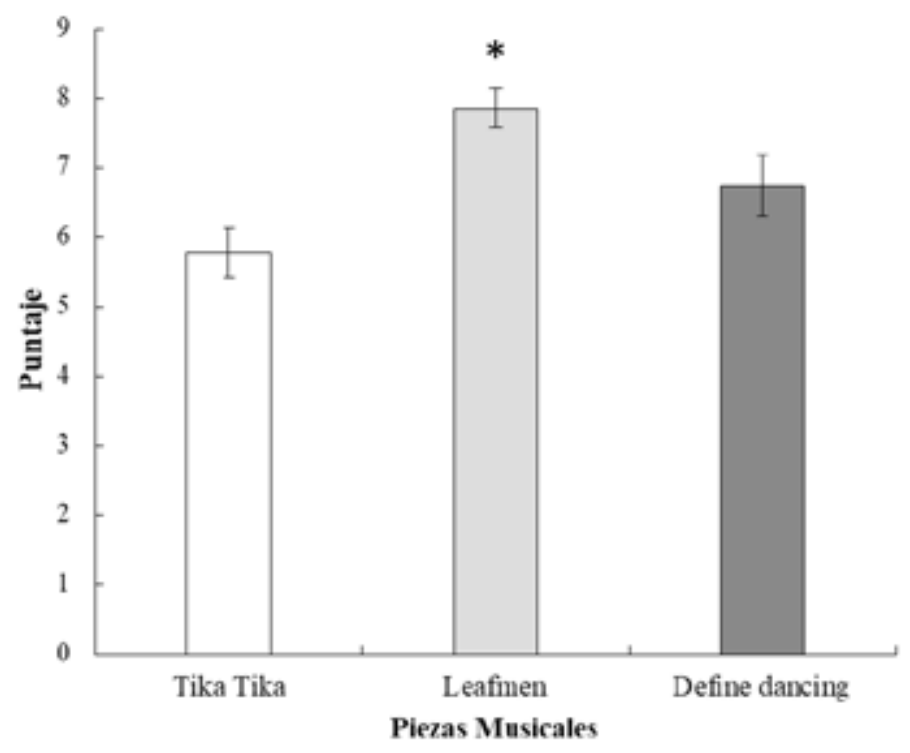

Figura 1. Comparación de las piezas musicales. Valoración del arousal (activación) de las tres piezas que escucharon los participantes. Las líneas verticales representan el error estándar. $* p<.05$, en comparación a las otras dos piezas oídas.

\section{Condición control (ruido blanco)}

Los sujetos escucharon ruido blanco con una intensidad media (señal aleatoria) por tres minutos (Rickard, Wing Wong y Velik, 2012). Esta condición estaba destinada a controlar posibles sesgos en los resultados debido a la percepción de un estímulo auditivo.

\section{Procedimiento}

El estudio se realizó en un único día de actividad, dividido en las siguientes fases (ver Figura 2 del diseño esquemático):

\section{Fase informativa}

Los participantes firmaron el consenti- 
miento informado y completaron el cuestionario sociodemográfico.

\section{Fase de inducción emocional}

Los participantes observaron las 24 imágenes y se les indicó que a medida que las observasen, consignasen en una planilla del 1 al 10 qué tan activantes les parecían cada una de ellas.

\section{Fase de medición (pre)}

Inmediatamente después de retirar la hoja de emocionalidad, se les entregaba la primera planilla de la EVEA para completar.

\section{Fase de percepción}

Al retirar la primera planilla de EVEA, se les indicaba que iban a escuchar un estímulo auditivo (pieza musical Leafmen para el grupo experimental o ruido blanco, para el grupo control) y que lo único que debían hacer era concentrarse en lo que estaban escuchando.

\section{Fase de medición (post)}

Finalizado el estímulo auditivo, se les entregaba la segunda planilla de la EVEA para completar.

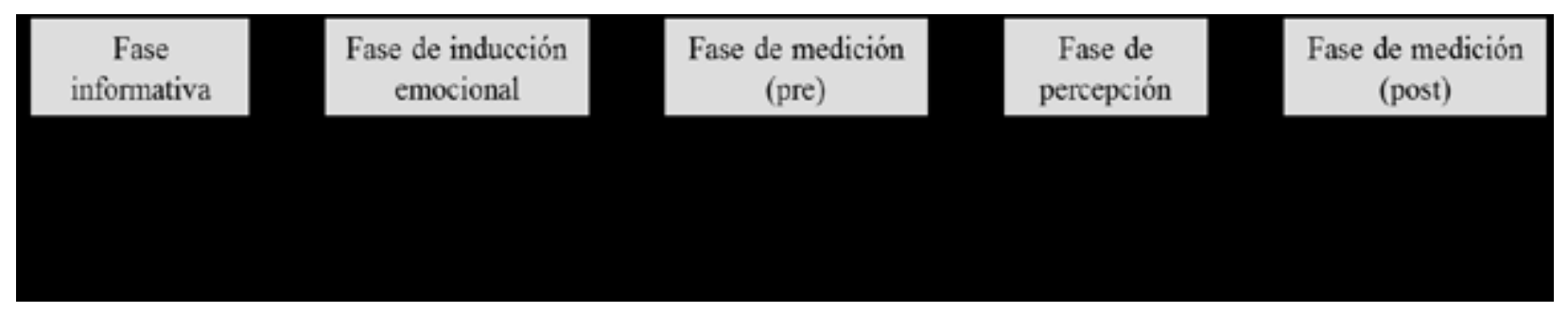

Figura 2. Diseño esquemático del estudio experimental

\section{Análisis de los datos}

En primera instancia se analizaron los supuestos de normalidad y homogeneidad de la muestra, para lo cual se utilizaron los estadísticos de Levenne y Shapiro Wilk. Debido a que la muestra no cumplía ambos supuestos, y ya que se trabajaron con los grupos en una medida de tipo pre-post tratamiento, se procedió a utilizar el test de Wilcoxon de muestras relacionadas para comparar cada grupo antes y después de la exposición a los estímulos auditivos. Se estableció un $p$ valor de .05 .

\section{Resultados}

\section{Arousal (nivel de activación)}

En una primera instancia los participantes debían observar una serie de imágenes con contenido negativo, con la finalidad de generar un estado emocional displacentero en los sujetos. A medida que observaban las imágenes debían calificar cuán activante les parecían en una escala de 1 a 10. Si bien en esta instancia los participantes aún no se encontraban divididos por tratamiento, se muestran los resultados de acuerdo a la posterior división realizada. No se hallaron diferencias en la valoración que les daban a las imágenes ambos grupos (datos no mostrados, $p>.05$ ).

\section{Estado de ánimo (Pretratamiento)}

Luego de observar las imágenes, los participantes completaron el EVEA. No se hallaron diferencias entre los grupos en esta instancia $(p>.05$; Figura 3$)$. 


\section{Estado de Ánimo (Postratamiento)}

Luego de la primera puntuación del EVEA, la mitad de los participantes fue asignado aleatoriamente al grupo control (sujetos que escucharon ruido blanco) o grupo experimental (sujetos que escucharon la pieza musical Leafmen). Se presentarán los resultados de la comparación de ambos EVEA (pre y post) para observar los datos antes y después de la intervención musical. Se separan los resultados de acuerdo a cada uno de los subítems para su correcta visualización (ver Figura 3).

\section{Tristeza}

En relación con el subítem Tristeza se observa que en el grupo control (expuesto a ruido blanco) no hay diferencia entre la puntuación que le otorgan a este ítem antes-después de la escucha del estímulo auditivo $(p>.05)$. Sin embargo, en el grupo experimental se observa una disminución en este ítem de la escala, dando cuenta de que la música fue efectiva modulando esta emoción $(\mathrm{Z}=-2.19, p=.03)$.

\section{Ansiedad}

En relación con el subítem Ansiedad se observa que no hay diferencias en el grupo

A

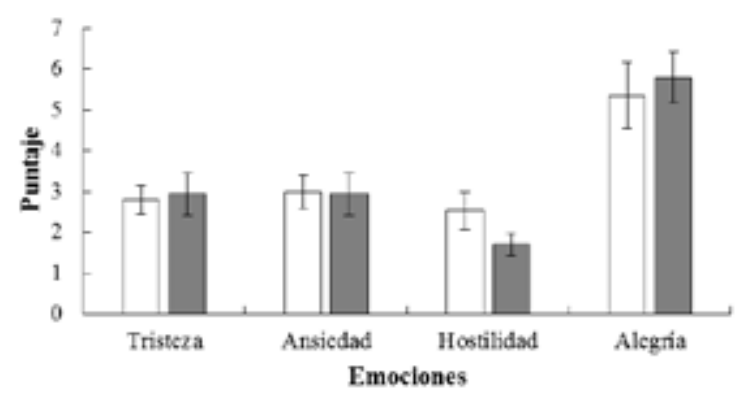

control (expuesto a ruido blanco) cuando se compara su puntuación antes y después del estímulo auditivo. Sin embargo, en relación al grupo experimental se observa que hay una disminución en este parámetro, nuevamente dando cuenta de la efectividad de la escucha de música activante para modular esta emoción $(\mathrm{Z}=2.72, p=.007)$.

\section{Hostilidad}

En relación con el subítem Hostilidad en ninguno de los dos grupos se observan cambios antes-después de la intervención auditiva $(p>.05)$.

\section{Alegría}

Por último, en relación con el subítem Alegría de la escala EVEA no se observan cambios antes-después en el grupo expuesto al ruido blanco $(p>.05)$. En el grupo experimental puede observarse un ascenso en este parámetro, sin embargo, los análisis estadísticos indican que no se llegó a la significación $(\mathrm{Z}=1.93, p=.053)$. Es probable que si se aumenta el tamaño de la muestra, esta tendencia a la significación se transforme en un resultado significativo. Es un posible camino para futuros estudios.

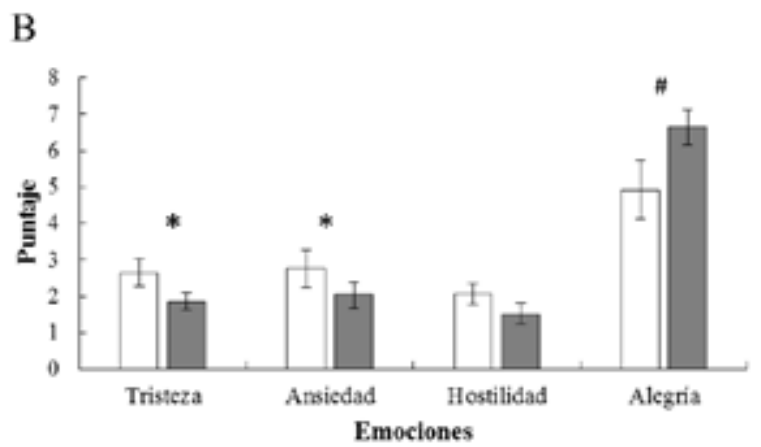

Figura 3. Puntuación que los participantes le dieron a cada uno de los ítems del EVEA. Los ítems se encuentran subdivididos en 4 emociones: Tristeza, Ansiedad, Hostilidad y Alegría. La barra blanca corresponde al Grupo Control y la barra gris, al Grupo Experimental. A: puntuaciones pretratamiento. Las líneas verticales representan el error estándar. B: puntuaciones postratamiento. Las líneas verticales representan el error estándar. ${ }^{*} p<.05$; $\# p=.053$ 


\section{Discusión}

El objetivo que guió el presente trabajo fue evaluar el efecto de la percepción musical con características activantes sobre el estado de ánimo de adultos jóvenes, para lo cual se indujo un estado emocional displacentero a través de la observación de imágenes con contenido emocional de valencia negativa. De acuerdo a los antecedentes sobre la temática, se predijo que la audición de un estímulo musical generaría una mejora en el estado emocional negativo de los sujetos.

Los resultados obtenidos señalan que la música especialmente seleccionada para los propósitos de este estudio sirvió como moduladora de las emociones de tristeza y ansiedad, patrón que no se observó con respecto al ítem Hostilidad. En relación con el ítem Alegría, hubo un aumento en el nivel emocional del grupo experimental, pero esta diferencia no llegó a la significación estadística. De esta forma se corrobora parcialmente la hipótesis inicialmente establecida.

La modulación de las emociones negativas a través de la percepción musical se justifica en el hecho de que la música posee la capacidad de inducir placer intenso y estimular los sistemas endógenos de la recompensa (Salimpoor et al., 2013). Estudios experimentales comprobaron que la música produce diferentes efectos neurofisiológicos que involucran la activación de los sistemas encargados del procesamiento emocional (Koelsch, 2009; Peretz et al., 1998). Estos datos sugieren que, aunque la música no sería imprescindible para la supervivencia de la especie humana, poseería un beneficio biológico significativo y generalmente subestimado en cuanto a su potencial para influir en las emociones y la conducta y, por lo tanto, contribuir al bienestar mental y físico (Chanda y Levitin, 2013).

La percepción musical es utilizada por diversas disciplinas (medicina, enfermería, musicoterapia, entre otras) como una herramienta dentro del conjunto de técnicas empleadas por profesionales de la salud y la educación. Sin embargo, muchos de los estudios experimentales realizados sobre la efectividad del uso de la música con fines que exceden la adquisición de habilidades musicales no han seguido reglas científicas rigurosas (Aldridge, 1994; Dumont, Syurina, Feron y van Hooren, 2017; Levitin, 2008; McConnell, Scott y Porter, 2016).

Una de las características más importantes de un experimento riguroso es el uso de la comparación o del control. Es por ello que los datos obtenidos mediante el presente estudio tienen relevancia científica en cuanto a que presentan indicios de la efectividad de la percepción musical para modular emociones, acompañando la creciente intención de generar investigaciones metodológicamente adecuadas (Poter et al., 2017), lo cual da lugar a hacer futuras investigaciones sobre musicoterapia receptiva para tratar trastornos relacionados con las emociones.

En cuanto a las limitaciones del estudio, y con nuevas perspectivas de trabajo a futuro, es importante no generalizar los resultados obtenidos a toda la población; en este sentido sería necesario ampliar la muestra a un $n$ mayor, así como también investigar este efecto en otras poblaciones (diferentes franjas etarias y diferentes niveles de entrenamiento musical, principalmente). Por otra parte, sería valioso estudiar este efecto con otros estímulos musicales, por ejemplo, con parámetros relajantes o con música elegida por los participantes, teniendo en cuenta, además, características psicosocioculturales de la muestra. Estos puntos serán contemplados y abordados en futuras líneas de investigación.

Finalmente, y en cuanto a las implicancias y aplicaciones del estudio realizado, los informes clínicos y los estudios realizados en pacientes que reciben tratamiento médico, en hospitales y otros centros de promoción de la salud, aportan datos que demuestran mayor eficacia del tratamiento cuando se controla el estado emocional del paciente (Taylor, 
2010). Muchos de estos informes señalan que la utilización apropiada de la música es un agente efectivo en la consecución de un estado emocional cooperativo, positivo $\mathrm{y}$ predecible. El empleo de estímulos musicales para producir emociones positivas cobra especial importancia en los casos en que lo principal sea apaciguar el estado emocional del individuo (Taylor, 2010). Por esta razón, es importante continuar estudiando de qué manera la música incide en las emociones, y así contribuir a una mejora en la salud no solo de los sujetos sanos, sino también de pacientes internados en hospitales $\mathrm{u}$ otras instituciones similares.

Los resultados obtenidos permiten también abrir nuevos caminos en cuanto a las cualidades intrínsecas de la música y sus elementos para promover alivio a la ansiedad y el estrés, regular estados de ánimo y humor, favorecer la motivación o posibilitar el desarrollo de mecanismos de adaptación a situaciones nuevas y/o traumáticas (Lichtensztejn, 2009). Finalmente, podría asumirse que la música constituye un estímulo efectivo para modular respuestas emocionales, que se ven reflejadas en los cambios ánimicos de los oyentes.

\section{Referencias bibliográficas}

Aldridge, D. (1994). An overview of music therapy research. Complementary Therapies in Medicine, 2(4), 204-216. http://dx.doi. org/10.1016/0965-2299(94)90021-3

Altenmüller, E. y Schlaug, G. (2015). Apollo's gift: new aspects of neurologic music therapy. Progress in Brain Research, 217, 237-52. http://dx.doi.org/10.1016/bs.pbr.2014.11.029

Asamblea Médica Mundial. (2013). Declaración de Helsinki de la AMM - Principios éticos para las investigaciones médicas en seres humanos. https://www.wma.net/es/policies-post/declaracion-de-helsinki-de-la-amm-principios-eticos-para-las-investigaciones-medicas-en-seres-humanos/

Blood, A. y Zatorre, R. (2001) Intensely pleasurable responses to music correlate with activity in brain regions implicated in reward and emotion. Proceedings of the National Academy of Science of the U.S.A., 98(20), 11818-11823. http://dx.doi.org/ 10.1073/pnas.191355898

Blood, A., Zatorre, R., Bermudez, P. y Evans, A. (1999). Emotional responses to pleasant and unpleasant music correlate with activity in paralimbic brain regions. Nature Neuroscience, 2, 382-387

Cahill, L., Gorski, L. y Le, K. (2003). Enhanced human memory consolidation with postlearning stress: Interaction with the degree of arousal at encoding. Learning \& Memory, 10(4), 270-274. http://dx.doi.org/ 10.1101/ $\operatorname{lm} .62403$

Chamorro-Premuzic, T. y Furham, A. (2007). Personality and music: Can traits explain how people use music in everyday life? British Journal of Psychology, 98(Pt. 2), 175-185. http://dx.doi.org/10.1348/000712606X111177

Chanda, M. y Levitin, D. (2013). The neurochemistry of music. Trends in Cognitive Sciences, 17(4), 179-193. http://dx.doi.org/ 10.1016/j. tics.2013.02.007.

Diaz Abrahan, V., Fisher, M. y Justel, N. (2017). Intervenciones musicoterapéuticas para la activación del sistema reticular. Interdisciplinaria, 34(1), 141-156. https://doi.org/10.16888/ interd.2017.34.1.9

Diaz Abrahan, V., Shifres, F. y Justel, N. (2018). Music improvisation modulates emotional memory. Psychology of Music, 48(4),1-16. http://dx.doi.org/ 10.1177/0305735618810793.

Díaz, J. (2010). Música, lenguaje y emoción: una aproximación cerebral. Salud Mental, 33, 543-551.

Dumont, E., Syurina, E. V., Feron, F. J. M. y van Hooren, S. (2017). Music interventions and child development: A critical review and further directions. Frontier in Psychology, 8(1694). http://dx.doi.org/10.3389/fpsyg.2017.01694

Fancourt, D., Ockelford, A. y Belai, A. (2014). The psychoneuroimmunological effects of music: A systematic review and a new model. Brain, Behavior, and Immunity, 36, 15-26. http://dx.doi.org/10.1016/j.bbi.2013.10.014

Flores-Gutierrez, E. y Díaz, J. (2009). La respuesta 
emocional a la música: atribución de términos de la emoción a segmentos musicales. Salud mental, 32, 21-34. https://www.redalyc.org/ articulo.oa?id=58212260004

Gosselin, N., Peretz, I., Johnsen, E. y Adolphs, R. (2007). Amygdala damage impairs emotion recognition from music. Neuropsychologia, 45, 236-244. https://doi.org/10.1016/j.neuropsychologia.2006.07.012

Gosselin, N., Peretz, I., Noulhiane, M., Hasboun, D., Beckett, C., Baulac, M. et al. (2005). Impaired recognition of scary music following unilateral temporal lobe excision. Brain, 128, 628-640. https://doi.org/10.1093/brain/awh420

Grocke, D., y Wigram, T. (2007). Receptive Methods in Music Therapy Techniques and Clinical Applications for Music Therapy Clinicians, Educators and Students. Philadelphia: Jessica Kingsley Publishers.

Juslin, P. N. y Sloboda, J. (2010). Handbook of Music and Emotion: Theory, Research, Applications. New York, USA: Oxford University Press.

Juslin, P. N., \& Västfjäll, D. (2008). Emotional responses to music: the need to consider underlying mechanisms. The Behavioral and brain sciences, 31(5), 559-621. https://doi. org/10.1017/S0140525X08005293

Justel, N. y Ruetti, E. (2014). Memoria emocional en adultos mayores: Evaluación del recuerdo de estímulos negativos. Cuadernos de Neuropsicologia/Panamerican Journal of Neuropsychology, 8(1), 107-119. http://www.cnps.cl/ index.php/cnps/article/view/158

Justel, N., O'Connor, J. y Rubinstein, W. (2015). Modulación de la memoria emocional a través de la música en adultos mayores: un estudio preliminar. Interdisciplinaria, 32(2), 247-259. https://doi.org/10.16888/interd.2015.32.2.3

Koelsch, S. (2009). A Neuroscientific Perspective on Music Therapy. The Neurosciences and Music III-Disorders and Plasticity: Annals of N.Y. Academy of Science. 374-384. http:// dx.doi.org/ 10.1111/j.1749-6632.2009.04592.x

Koelsch, S., Fritz, T., V Cramon, D. Y., Müller, K., y Friederici, A. D. (2006). Investigating emotion with music: an fMRI study. Human brain mapping, 27(3), 239-250. https://doi. org $/ 10.1002 / \mathrm{hbm} .20180$

Koelsch, S., Skouras, S. y Lohmann, G. (2018). The auditory cortex hosts network nodes influential for emotion processing: An fMRI study on music-evoked fear and joy. Plos One, 13, 22. http://dx.doi.org/10.1371/journal. pone. 0190057

Kreutz, G., Ott, U., Osawa, P., Teichmann, P.y Vaitl, D. (2008). Using music to induce emotions: Influences of musical preference and absorption. Psychology of Music, 36(1), 101-126. http://dx.doi.org/10.1177/0305735607082623

Lang, P. J., Bradley, M. M. y Cuthbert, B. N. (1995). International affective picture system (IAPS): affective ratings of pictures and instruction manual. Technical Report A-6. Gainesville, FL: University of Florida.

Levitin, D. (2008). El cerebro musical. Seis canciones que explican la evolución humana. Barcelona: RBA.

Lichtensztejn, M. (2009). Música \& Medicina. La aplicación especializada de la música en el área de la salud. Argentina: Ediciones Elemento.

McConnell, T., Scott, D. y Porter, S. (2016). Music therapy for end-of-life care: an updated systematic review. Palliative Medicine, 30(9), 877-83. http://dx.doi.org/10.1177/0269216316635387.

Peretz, I., Gagnon, L. y Bouchard, B. (1998). Music and emotion: Perceptual determinants, immediacy, and isolation after brain damage. Cognition, 68(1), 111-141. doi: 10.1016/j. cognition.2005.11.004

Porter, S., McConnell, T., Clarke, M., Kirkwood, J., Hughes, N., Graham-Wisener, L., Regan, J., McKeown, M., McGrillen, K., \& Reid, J. (2017). A critical realist evaluation of a music therapy intervention in palliative care. BMC palliative care, 16(1), 70. https://doi. org/10.1186/s12904-017-0253-5

Radford, C. (1989). Emotions and music: A reply to the cognitivists. The Journal of Aesthetics and Art Criticism, 47, 69-76. http://dx.doi. org/10.2307/431994

Rickard, N. (2004) Intense emotional responses to music: a test of the physiological arousal 
hypothesis. Psichology of Music, 32(4), 371-388.

Rickard, N., Wing Wong, W. y Velik, L. (2012). Relaxing music counters heightened consolidation of emotional memory. Neurobiology of Learning \& Memory, 97(2), 220-228. http:// dx.doi.org/10.1016/j.nlm.2011.12.005.

Saarikallio, S. y Erkkilä, J. (2007). The role of music in adolescents' mood regulation. Psychology of Music, 35(1), 88-109. http:// dx.doi.org/10.1177/0305735607068889

Salimpoor, V. N., van den Bosch, I., Kovacevic, N., McIntosh, A. R., Dagher, A. y Zatorre, R. J. (2013). Interactions between the nucleus accumbens and auditory cortices predict music reward value. Science, 340, 216-219. http:// dx.doi.org/10.1126/science. 1231059 .

Sanz, J. (2001). Un instrumento para evaluar la eficacia de los procedimientos de inducción de estado de ánimo: La Escala de Valoración del Estado de ánimo (EVEA). Análisis y modificación de la conducta, 111(27), 71-110. http:// www.uhu.es/publicaciones/ojs/index.php/amc/ index

Silva, J. (2005). Regulación emocional y psicopatología: el modelo de vulnerabilidad/resiliencia. Revista Chilena de Neuro-Psiquiatría, 43(3), 201-209. http://dx.doi.org/10.4067/ S0717-92272005000300004

Sloboda, J. y O’Neill, S. A. (2001). Emotions in everyday listening to music. En P. Juslin y J. Sloboda, Music and emotion: Theory and research (pp. 415-430). New York, USA: Oxford University Press

Taylor, D. (2010). Fundamentos biomédicos de la musicoterapia. Bogotá: Cedro Impresores.

Thurston, H., Bell, J. e Induni, M. (2018). Community-level adverse experiences and emotional regulation in children and adolescents. Journal of Pediatric Nursing, 42, 25-33. http://dx.doi. org/10.1016/j.pedn.2018.06.008

Von Georgi, R. y Polat, B. (2013). Emotion, personality, Use of music in everyday life and musical preferences. Proceedings of the $3^{\text {rd }}$ International Conference on Music Y Emotion.

Recibido: 15 de febrero de 2019 Aceptado: 23 de septiembre de 2020 
